\title{
Analysis of a two-phase model describing the growth of solid tumors
}

\author{
JOACHIM ESCHER and ANCA-VOICHITA MATIOC \\ Institute for Applied Mathematics, Leibniz University Hanover, Welfengarten 1, 30167 Hanover, Germany \\ emails: escher@ifam.uni-hannover.de, matioca@ifam.uni-hannover.de
}

(Received 22 October 2010; revised 23 August 2012; accepted 23 August 2012;

first published online 19 September 2012)

\begin{abstract}
In this paper we consider a two-phase model describing the growth of avascular solid tumors when taking into account the effects of cell-to-cell adhesion and taxis due to nutrient. The tumor is surrounded by healthy tissue which is the source of nutrient for tumor cells. In a three-dimensional context, we prove that the mathematical formulation corresponds to a well-posed problem, and find radially symmetric steady-state solutions of the problem. They appear in the regime where the rate of cell apoptosis to cell proliferation is less than the far field nutrient concentration. Furthermore, we study the stability properties of those radially symmetric equilibria and find, depending on the biophysical parameters involved in the problem, both stable and unstable regimes for tumor growth.
\end{abstract}

Key words: Radially symmetric stationary solution; Classical solution; Stability; Tumor growth; Taxis

\section{Introduction}

Most of the tumor models considered in the mathematical literature have the hindrance of considering the evolution of the tumor without taking into account the influence of the healthy tissue surrounding the tumor on its growth. Multi-phase tumor models consider the tumor as being a saturated medium comprising at least one solid and one liquid phase, the behaviour at any point being influenced by mechanisms in several different phases: the extracellular fluid, the extracellular matrix or different cell types. These models arise, therefore, as a natural improvement of single-models and allow one to incorporate more of the biophysical processes which are relevant for the tumor evolution. Their complexity though limits in most of the cases the analysis and numerical studies to consider one-dimensional or radially symmetric evolution, cf. [2,10]. As observed in [11] and [12], radial symmetric tumor evolution may not always reflect the complexity and may also create a false impression about the features of the model: There exist radially symmetric steady states of the one-phase model analyzed in [11] and [12] which are unstable, but attract at an exponential rate radially symmetric solutions.

In this paper we analyze a two-phase tumor model proposed recently in [3]. Particular features of this model are the facts that the tumor evolution is coupled to the evolution of the healthy tissue surrounding it, and the inclusion of cell-to-cell adhesion and nutrient induced taxis. The surrounding tissue is treated as an inviscid fluid, whereas the tumor 
cells behave like a viscous fluid, both having the same density. Moreover, the nutrient concentration $\sigma_{\infty}$ is assumed constant in the far field tissue. The nutrient acts as a chemo-attractant for tumor cells, and the taxis is neglected in the tissue region. The model describes the evolution of the nutrient (e.g. oxygen or glucose), which is consumed proportionally to the local nutrient concentration, and a modified pressure coupled to that of the tumor boundary.

In [3] the authors obtain for the first time a Cahn-Hilliard-type model for a twocomponent mixture of tumor tissue and water. The mass exchange between the two phases is due to cell-mitosis in the tumor component at a rate proportional to the nutrient concentration, and the tumor mass is converted into fluid due to cell apoptosis. The relevant non-dimensionalized parameters appearing in this model (and which are relevant for our analysis) are

$$
\mathscr{G}:=\frac{l \bar{\chi}_{\sigma} \sigma_{\infty}}{\varepsilon \sqrt{\bar{f} \phi_{T}}}, \quad \mathscr{P}:=\frac{\lambda_{p} \sigma_{\infty}}{\lambda_{\chi}}, \quad \mathscr{A}:=\frac{\lambda_{A}}{\lambda_{\chi}}, \quad l:=\sqrt{D_{T} / \lambda_{\sigma} \phi_{T}}, \quad \lambda_{\chi}:=\frac{M \bar{\chi}_{\sigma} \sigma_{\infty} \phi_{T}}{l^{2}},
$$

where $\varepsilon$ measures the strength of tumor-tissue interaction, $\lambda_{p}$ is the proliferation rate per unit mass, $\lambda_{A}$ is the apoptosis rate per unit mass, $\bar{f}$ is a characteristic interaction energy, $D_{T}$ is the diffusion coefficient in the tumor, $\Phi_{T}$ is the characteristic solid tumor fraction, $\bar{\chi}_{\sigma}$ is the characteristic taxis coefficient, $M$ is a permeability matrix and $\lambda_{\sigma}$ is the nutrient uptake rate. Then, assuming a smooth transition layer between the tumor and the water (or healthy tissue domain), by a matched asymptotic analysis, the authors arrive at the following two-phase moving boundary problem:

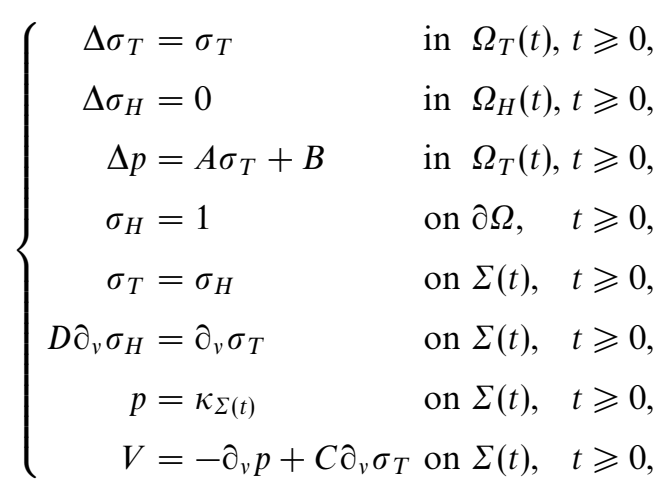

where $\Omega_{T}(t)$ is the tumor domain and $\Omega_{H}(t)$ is the surrounding tissue at time $t$. These two phases are separated by a sharp interface $\Sigma(t), \Omega_{T}(t)$ being the interior region of the fixed domain $\Omega \subset \mathbb{R}^{3}$. The functions $\sigma_{H}$ and $\sigma_{T}$ denote the non-dimensionalized nutrient concentration within $\Omega_{H}$ and $\Omega_{T}$, respectively, while $p$ is a modified pressure. Together with the interior boundary $\Sigma(t)$ they are the unknowns of the problem. The mean curvature, the normal velocity of $\Sigma(t)$ and the outward normal at $\partial \Omega_{T}(t)=\Sigma(t)$ are denoted by $\kappa_{\Sigma(t)}, V$, and $v$ respectively.

The system (1.2) is supplemented by the initial condition

$$
\Sigma(0)=\Sigma_{0}
$$


where $\Sigma_{0}$ is the boundary of a (sufficiently smooth) simply connected domain $\Omega_{T 0}$ contained in $\Omega$. The constants $A, B, C$ and $D$ are related to those defined by (1.1) as follows:

$$
A:=\tilde{\mathscr{G}}\left(\chi_{\sigma}-\mathscr{P}\right), \quad B:=\tilde{\mathscr{G}} \mathscr{A}, \quad C:=\tilde{\mathscr{G}}_{\chi_{\sigma}}, \quad D:=\frac{D_{H}}{D_{T}},
$$

where $D_{H}$ is the diffusion coefficients in the healthy tissue, $\tilde{\mathscr{G}}$ is a positive constant which incorporates the effect of cell-to-cell adhesion and $\chi_{\sigma}$ is the non-dimensionalized taxis coefficient, cf. [3]. The natural restriction we make in this paper is that $C-A>0$ (this is, cf. (1.4), the biologically relevant situation). This model describes the evolution of a solid avascular tumor, located at $\Omega_{T}$, which is surrounded by healthy tissue $\Omega_{H}$.

It should be noted that this model is an extension (taxis is taken into account) of previous single phase models [1,4], where the driving mechanisms were the pressure and cell proliferation. The moving boundary problems associated to the models presented in $[1,4]$ have been investigated by many authors (see $[5-9,11,12,19-21]$ and the references therein). Unlike the models studied in $[10,13,23,24]$ where existence of a necrotic core consisting of death cells is taken into account, the model derived in [3] and studied herein does not possess this feature. However, system (1.2) has two phases, and the nutrient concentrations $\sigma_{T}$ and $\sigma_{H}$, inside and outside the solid tumor, are connected through a diffraction problem, a feature which relates system (1.2) to the Muskat problem, cf. $[14,15,22]$.

We show in Theorem 3.1 that all these new aspects considered in (1.2) do not influence the well-posedness of the problem. For initial data close to an arbitrary smooth domain, we show that the solution of (1.2) and (1.3) exists atleast locally. To this scope we transform the problem into an abstract parabolic evolution equation and use a theorem from [25] to prove existence and uniqueness of classical solutions. Furthermore, it turns out that problem (1.2) possesses radially symmetric stationary solutions only if the rate of cell apoptosis to cell proliferation is less than the far field nutrient concentration. Unlike the model proposed in [4], where the radius of the stationary tumor depends only of one parameter, the situation considered here is more involved and the radii of the steady states depend on all parameters appearing in problem (1.2), a feature which reflects the complexity of the model.

The stability properties of these special solutions are analyzed in Theorems 4.1 and 4.3. We show that unlike [7], where a threshold value for the surface tension coefficient of the tumor boundary was found to distinguish between stable and unstable growth regimes, the situation herein is more involved and the stability of spherical equilibria is determined by a plethora of factors. For example, if the diffusion coefficient in the tumor tissue is larger than that in the healthy tissue, and the regions rich in nutrients are closed to the tumor, then the equilibria are unstable. This fits with the numerical simulations presented in [3] for the mixture model where it is shown in a two-dimensional context that initially avoids tumors that penetrate the healthy tissue by developing invasive fingers. Moreover, we show that there exists also a certain range of the parameters $A, B, C, D$, where, starting close to the steady state, the tumor evolves exponentially fast towards this equilibrium, a feature which is not captured in [3].

Finally, let us observe that if the nutrient concentration $\sigma_{H}$ is constant in $\Omega_{H}(t)$, the taxis is absent, $C=0$, and we drop the sixth equation of (2.1) to arrive at the one-phase tumor 
model analyzed in $[6,8,20]$. While in $[8,20]$ the equations coincide with those remaining in (2.1), the authors in [6] consider the problem of existence of non-radial stationary solution for the model when the first and the third equation of (2.1) are replaced with the more general relations

$$
\left\{\begin{array}{rl}
\Delta \sigma_{T} & =f\left(\sigma_{T}\right) \text { in } \Omega_{T}, t \geqslant 0 \\
\Delta p & =g\left(\sigma_{T}\right) \text { in } \Omega_{T}, t \geqslant 0
\end{array},\right.
$$

the mappings $f$ and $g$ being the nutrient consumption rate function and the tumor cell proliferation rate function respectively. For our two-phase model though, if $f$ has a general form, in order to prove the well-posedness of the problem we need to solve the semi-linear diffraction problem (2.1) with the first equation replaced by the corresponding equation of (1.5). As far as we know, the existence of solutions for this type of problems has not been investigated yet, so that one has to restrict to $f$ being linear. On the other hand, we may assume the function $g$ to have a general form in our case. Provided merely $g \in C^{\infty}(\mathbb{R})$, our analysis shows that the corresponding two-phase model is well-posed. However, determining the existence of radially symmetric steady-state solutions (and studying their stability properties) under suitable conditions on $g$ is mathematically much more challenging in this case.

The outline of the paper is as follows. We study in Section 2 the radially symmetric case when $\Omega$ is itself a ball, and prove that the system (1.2) possesses spherically stationary solutions, result established in Theorem 2.1. In Section 3 we prove the well-posedness of the problem, and the last section is dedicated to the study of the stability properties of the stationary solutions identified in Section 2.

\section{Radially symmetric solutions}

We first note that the set of solutions of (1.2) is invariant under rotations and translations in $\mathbb{R}^{3}$. Therefore, we shall consider in the remainder of this paper that the domain $\Omega$ contains the origin of $\mathbb{R}^{3}$.

In this section we are concerned with the question whether there exist radially symmetric steady states of problem (1.2), i.e. we look for positive real numbers $R_{1}$ and $R_{2}$ with $R_{1}<R_{2}$ such that $\Omega_{T}=\mathbb{B}\left(0, R_{1}\right)$, and the annulus $\Omega_{H}:=\mathbb{A}\left(R_{1}, R_{2}\right):=\left\{y \in \mathbb{R}^{3}: R_{1}<|y|<R_{2}\right\}$ is a solution of (1.2). Of course, the existence of this solution may also depend on the parameters $A, B, C, D$ involved in the modeling. To this scope we first consider the diffraction problem,

$$
\left\{\begin{aligned}
\Delta \sigma_{T}-\sigma_{T}=0 & \text { in } \Omega_{T}, \\
\Delta \sigma_{H}=0 & \text { in } \Omega_{H}, \\
\sigma_{H}=1 & \text { on } \partial \Omega, \\
\sigma_{T}-\sigma_{H}=0 & \text { on } \Sigma, \\
D \partial_{v} \sigma_{H}-\partial_{v} \sigma_{T}=0 & \text { on } \Sigma,
\end{aligned}\right.
$$

where $\Omega_{H}$ is assumed to be a $C^{2+\alpha}$-domain, $\alpha \in(0,1)$ and $D>0$. We claim that this problem has a unique solution $\left(\sigma_{T}, \sigma_{H}\right)$ within the class $B U C^{2+\alpha}\left(\Omega_{T}\right) \times B U C^{2+\alpha}\left(\Omega_{H}\right)$ consisting of functions having the uniformly Hölder continuous second order derivatives. 
Since from [25, Theorems 16.1 and 16.2] the existence of a classical solution to the divergence problem (2.1) is implied by the uniqueness of the solution to (2.1), it suffices to show that the system has, when the right-hand side of its third equation is a constant 0 , only the trivial solution $\left(\sigma_{T}, \sigma_{H}\right)=(0,0)$. Let us assume the contrary. The function $\sigma:=\sigma_{T} \chi_{\Omega_{T}}+\sigma_{H} \chi_{\Omega_{H}}$, where $\chi_{M}$ stands for the characteristic function of $M \subset \mathbb{R}^{3}$, is uniformly continuous in $\Omega$ so that we may presuppose that there exists a point $x_{0} \in \Omega$ such that $\sigma\left(x_{0}\right)=\max _{\Omega} \sigma>0$. The strong elliptic maximum principle ensures that this point belongs to $\Sigma$. Hence, $\sigma_{H}$ is not constant and the Hopf's lemma ensures that $\partial_{v} \sigma_{H}<0$ ( $v$ points into $\Omega_{H}$ ). We conclude that $\partial_{v} \sigma_{T}<0$, which contradicts Hopf's lemma.

Summarising, (2.1) has a unique solution, and since the right-hand sides of equations in (2.1) are constant, this solution depends only on the radial coordinate $r \in\left[0, R_{2}\right]$ if $\Omega_{T}=\mathbb{B}\left(0, R_{1}\right)$ and $\Omega_{H}=\mathbb{A}\left(R_{1}, R_{2}\right)$. Using spherical coordinates, we may determine $\left(\sigma_{T}, \sigma_{H}\right)$ if we solve the following system:

$$
\left\{\begin{array}{rlrl}
\sigma_{T}^{\prime \prime}(r)+2 r^{-1} \sigma_{T}^{\prime}(r)-\sigma_{T}(r) & =0, & & 0<r<R_{1}, \\
\sigma_{H}^{\prime \prime}(r)+2 r^{-1} \sigma_{H}^{\prime}(r) & =0, & & R_{1}<r<R_{2}, \\
\sigma_{H}\left(R_{2}\right) & =1, & \\
\sigma_{T}\left(R_{1}\right) & =\sigma_{H}\left(R_{1}\right), & \\
D \sigma_{H}^{\prime}\left(R_{1}\right) & =\sigma_{T}^{\prime}\left(R_{1}\right) . &
\end{array}\right.
$$

Using the first equation, respectively the second and the third, we determine the following explicit relations for $\sigma_{T}$ and $\sigma_{H}$ :

$$
\sigma_{T}(r)=\alpha \frac{\sinh (r)}{r} \quad \text { and } \quad \sigma_{H}(r)=1-\frac{\beta}{R_{2}}+\frac{\beta}{r}
$$

with constants $\alpha$ and $\beta$ chosen such that $\sigma_{T}$ and $\sigma_{H}$ also verify the last two equations of the system, that is

$$
\begin{gathered}
\alpha:=\frac{D R_{1} R_{2}}{D R_{2} \sinh \left(R_{1}\right)+\left(R_{2}-R_{1}\right)\left(R_{1} \cosh \left(R_{1}\right)-\sinh \left(R_{1}\right)\right)}, \\
\beta:=-\frac{R_{1} R_{2}\left(R_{1} \cosh \left(R_{1}\right)-\sinh \left(R_{1}\right)\right)}{D R_{2} \sinh \left(R_{1}\right)+\left(R_{2}-R_{1}\right)\left(R_{1} \cosh \left(R_{1}\right)-\sinh \left(R_{1}\right)\right)} .
\end{gathered}
$$

With $\sigma_{T}$ given by (2.3) and (2.4), we may proceed and determine the pressure by solving the Dirichlet problem

$$
\left\{\begin{aligned}
p^{\prime \prime}(r)+2 r^{-1} p^{\prime}(r) & =\alpha A r^{-1} \sinh (r)+B, 0<r<R_{1}, \\
p\left(R_{1}\right) & =R_{1}^{-1},
\end{aligned}\right.
$$

which is obtained by rewriting the third and seventh equations of (1.2) in spherical coordinates and by taking into account that both $\sigma_{T}$ and $\kappa_{\Sigma}$ depend only upon $r$ when 
$\Omega_{T}=\mathbb{B}\left(0, R_{1}\right)$. Multiplying the second equation of (2.6) by $r^{2}$ and integrating, we obtain

$$
\begin{aligned}
r^{2} p^{\prime}(r)= & \alpha A(r \cosh (r)-\sinh (r))+\frac{B r^{3}}{3}- \\
& \alpha A\left(R_{1} \cosh \left(R_{1}\right)-\sinh \left(R_{1}\right)\right)-B \frac{R_{1}^{3}}{3}+R_{1}^{2} p^{\prime}\left(R_{1}\right)
\end{aligned}
$$

for all $r \in\left[0, R_{1}\right]$. Moreover, since $p \in C^{\infty}\left(\left[0, R_{1}\right]\right)$, we get

$$
p^{\prime}(r)=\alpha A \frac{r \cosh (r)-\sinh (r)}{r^{2}}+\frac{B r}{3} .
$$

This last relation and the second equation of (2.6) lead us to the following expression for $p$ :

$$
p(r)=\frac{1}{R_{1}}-\alpha A \int_{r}^{R_{1}} \frac{s \cosh (s)-\sinh (s)}{s^{2}} d s+\frac{B}{6} r^{2}-\frac{B R_{1}^{2}}{6} .
$$

Finally, $\Omega_{T}=\mathbb{B}\left(0, R_{1}\right)$ and $\Omega_{H}=\mathbb{A}\left(R_{1}, R_{2}\right), R_{1}<R_{2}$, is a stationary solution of problem (1.2) if $p, \sigma_{T}$ and $\sigma_{H}$ are given by (2.3) and (2.7), respectively, and if the last relation of (1.2) is fulfilled. Hence, we have to verify that $p^{\prime}\left(R_{1}\right)=C \sigma_{T}^{\prime}\left(R_{1}\right)$, a relation which is equivalent to the following equation:

$$
B=3 \alpha(C-A) \frac{R_{1} \cosh \left(R_{1}\right)-\sinh \left(R_{1}\right)}{R_{1}^{3}} .
$$

We use (2.8) to express $R_{2}$ in dependence of $A, B, C, D$ and $R_{1}$, because we still have to verify that $R_{1}<R_{2}$. Combining (2.4) and (2.8), we get

$$
R_{2}=\frac{B R_{1}^{3}\left(R_{1} \cosh \left(R_{1}\right)-\sinh \left(R_{1}\right)\right)}{\left[B R_{1}^{2}-3(C-A) D\right]\left(R_{1} \cosh \left(R_{1}\right)-\sinh \left(R_{1}\right)\right)+B D R_{1}^{2} \sinh \left(R_{1}\right)},
$$

thus $R_{2}>R_{1}$ if and only if

$$
\frac{R_{1}^{2}}{D}+\frac{R_{1}^{2} \sinh \left(R_{1}\right)}{R_{1} \cosh \left(R_{1}\right)-\sinh \left(R_{1}\right)}>\frac{3(C-A)}{B}>\frac{R_{1}^{2} \sinh \left(R_{1}\right)}{R_{1} \cosh \left(R_{1}\right)-\sinh \left(R_{1}\right)} .
$$

The first inequality is obtained by imposing that the denominator of (2.9) is positive (the numerator is obviously positive), while the second one is exactly the condition $R_{2}>R_{1}$. In order to present our first main result, we consider, motivated by (2.10), the auxiliary function $f:[0, \infty) \rightarrow \mathbb{R}$ given by

$$
f(x):=\frac{x^{2} \sinh (x)}{x \cosh (x)-\sinh (x)}, \quad x \in[0, \infty) .
$$

We first claim that $f$ is strictly increasing. Indeed, given $x \geqslant 0$, we compute that $f^{\prime}(x)=x g(x) /(x \cosh (x)-\sinh (x))^{2}$, whereby

$$
g(x):=x^{2}+x \sinh (x) \cosh (x)-2 \sinh ^{2}(x) .
$$


The function $g$ has the property that $g^{(m)}(0)=0$ for all $m \leqslant 2$, while

$$
g^{(3)}(x)=4 x \sinh ^{2}(x)+4 \cosh (x)(x \cosh (x)-\sinh (x)) \geqslant 0
$$

for all $x \geqslant 0$. Consequently, $f$ is a strictly increasing function. Since $f(0)=3$, we obtain the following restriction for the ratio $(C-A) / B$, namely

$$
\frac{C-A}{B}>1
$$

Thus, given positive constants $A, B, C, D$ with $(C-A) / B>1$, there exist unique positive real numbers $R_{1 *}<R_{1}^{*}$ such that

$$
\frac{R_{1^{*}}^{2}}{D}+\frac{R_{1^{*}}^{2} \sinh \left(R_{1^{*}}\right)}{R_{1^{*}} \cosh \left(R_{1 *}\right)-\sinh \left(R_{1 *}\right)}=\frac{3(C-A)}{B}=\frac{R_{1}^{* 2} \sinh \left(R_{1}^{*}\right)}{R_{1}^{*} \cosh \left(R_{1}^{*}\right)-\sinh \left(R_{1}^{*}\right)}
$$

Since (2.10) is satisfied by $R_{1}$ exactly when $R_{1} \in\left(R_{1 *}, R_{1}{ }^{*}\right)$, we conclude this section with the following result.

Theorem 2.1 (Radially symmetric equilibria) Let $A \in \mathbb{R}$ and let $B, C, D$ be positive constants and assume that (2.11) is satisfied. Then $\left(\Omega_{T}, \Omega_{H}\right):=\left(\mathbb{B}\left(0, R_{1}\right), \mathbb{A}\left(R_{1}, R_{2}\right)\right)$ with $R_{1} \in\left(R_{1 *}, R_{1}{ }^{*}\right)$ and $R_{2}$ given by (2.9) is a stationary solution of (1.2).

Moreover, there exists no other radially symmetric stationary solutions of (1.2).

Remark 2.2 Note that the above condition (2.11) is satisfied exactly when,

$$
\frac{\lambda_{A}}{\lambda_{p}}<\sigma_{\infty}
$$

cf. (1.1) and (1.4), showing that there must exist a balance between the rate of mitosis, the rate of apoptosis and the far field nutrient concentration. Moreover, the exterior radius $R_{2} \rightarrow \infty$ for $R_{1} \rightarrow R_{1^{*}}$, while for $R_{1} \rightarrow R_{1}^{*}$ we have that $R_{2} \rightarrow R_{1}^{*}$.

\section{The local well-posedness result}

In this section we are concerned with the problem whether the system (1.2) is well-posed for arbitrary initial data. To do this, we assume that the tumor evolves within the domain $\Omega$, which is presupposed to be smooth. Furthermore, let $\Sigma$ be the boundary of a bounded and simply connected smooth domain $\Omega_{T} \subset \Omega$ and pick $a<\operatorname{dist}(\Sigma, \partial \Omega)$. Denoting by $v$ the outward unit normal at $\Sigma$, we proceed as in $[17,18]$ and define the mapping

$$
X: \Sigma \times(-a, a) \rightarrow \mathbb{R}^{3}, \quad(x, \lambda) \mapsto x+\lambda v(x),
$$

which is a smooth diffeomorphism from $\Sigma \times(-a, a)$ onto its image $\mathscr{R}:=\operatorname{im}(X)$, i.e. $X \in \operatorname{Diff}^{\infty}(\Sigma \times(-a, a), \mathscr{R})$. We decompose the inverse of $X$ into $X^{-1}=(P, \Lambda)$ with both $P$ and $\Lambda$ being smooth. Here $P$ is the projection from $\mathscr{R}$ on $\Sigma$, i.e. $P(y)$ is the nearest point on $\Sigma$ to $y$ and $\Lambda$ is the signed distance from $y$ to $\Sigma$ (that is to $P(y)$ ). We will parametrise 
the solid tumor by using functions from

$$
\text { Ad }:=\left\{\rho \in C^{2}(\Sigma):\|\rho\|_{C(\Sigma)}<a / 4\right\}
$$

which we call to be a set of admissible functions. More precisely, we associate to each $\rho \in \mathrm{Ad}$, a unique $C^{2}$-surface $\Sigma(\rho)$, namely the image of the function $\theta_{\rho}(x):=x+\rho(x) v(x)$, $x \in \Sigma$. Indeed, since $|\rho(x)|<a / 4$ for all $x \in \Sigma$, we get with our choice of $a$ that $\theta_{\rho} \in \operatorname{Diff}^{2}(\Sigma, \Sigma(\rho))$. Note that $y \in \Sigma(\rho)$ if and only if $\Lambda(y)=\rho(P(y))$, meaning that $\Sigma(\rho)=\mathscr{N}_{\rho}^{-1}(0)$, where $\mathscr{N}_{\rho}: \mathscr{R} \rightarrow \mathbb{R}$ is defined by

$$
\mathscr{N}_{\rho}(y)=\Lambda(y)-\rho(P(y)) \text { for } y \in \mathscr{R} \text {. }
$$

Therefore, $v_{\rho}:=\nabla \mathscr{N}_{\rho} /\left|\nabla \mathscr{N}_{\rho}\right|$ is the outward-orientated unit normal at $\Sigma(\rho)$. Indeed, for $h$ being small enough, we have

$$
\mathscr{N}_{\rho}(x+(\rho(x)+h) v(x))-\mathscr{N}_{\rho}(x+\rho(x) v(x))=h,
$$

thus $\left\langle\nabla \mathscr{N}_{\rho}(x+\rho(x) v(x)) \mid v(x)\right\rangle=1$ for all $x \in \Sigma$. Here and in the following $\langle\cdot \mid \cdot\rangle$ stands for the scalar product in $\mathbb{R}^{3}$. Presuppose now that $\rho:[0, T] \rightarrow \mathrm{Ad}, T>0$, describes the evolution of the boundary separating the tumor and the host domain, i.e. $\Sigma(t)=\Sigma(\rho(t))$ for all $t \in[0, T]$. Then the solid tumor is located at $\Omega_{T}(\rho(t))$, the connected component of $\mathbb{R}^{3}$ bounded by $\Sigma(\rho(t))$, while the host domain is $\Omega_{H}(\rho(t)):=\Omega \backslash \bar{\Omega}_{T}(\rho(t))$ for each $t \in[0, T]$. Using the function $\mathscr{N}_{\rho}$, the normal velocity of $\Sigma(\rho(t))$ is given by the relation

$$
V=\frac{\partial_{t} \rho(t)}{\left|\nabla \mathscr{N}_{\rho(t)}\right|} \text { on } \Sigma(\rho(t)), t \in[0, T]
$$

With this notation, we may rewrite our problem (1.2)-(1.3) as nonlinear and non-local problem having also $\rho$ as an unknown,

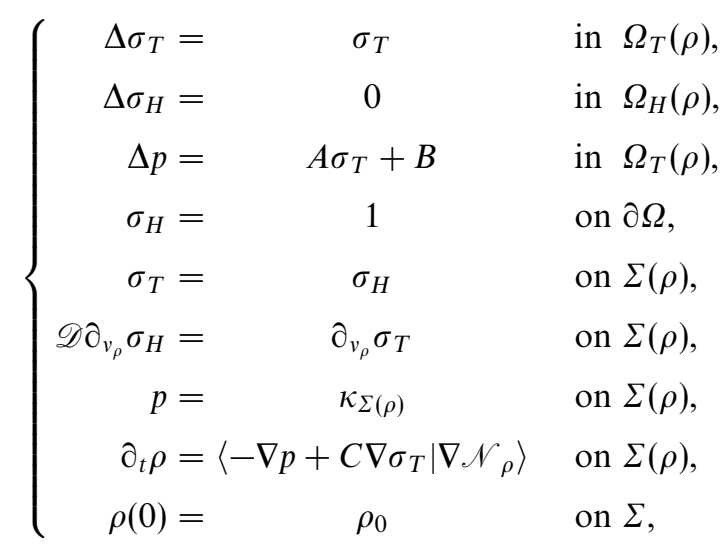

where $t \in[0, T]$ and $\rho_{0}$ is the initial shape of the tumor. Let $\alpha \in(0,1)$ be fixed for the remainder of this paper. A tuple $\left(\rho, \sigma_{H}, \sigma_{T}, p\right)$ is called the classical Hölder solution of 
(3.1) if

$$
\begin{aligned}
& \rho \in C\left([0, T], h^{4+\alpha}(\Sigma) \cap \mathrm{Ad}\right) \cap C^{1}\left([0, T], h^{1+\alpha}(\Sigma)\right), \\
& \sigma_{T}(t, \cdot), p(t, \cdot) \in b u c^{2+\alpha}\left(\Omega_{T}(\rho(t))\right), \quad \sigma_{H}(t, \cdot) \in b u c^{2+\alpha}\left(\Omega_{H}(\rho(t))\right) \text { for all } t \in[0, T],
\end{aligned}
$$

and $\left(\rho, \sigma_{H}, \sigma_{T}, p\right)$ solves (3.1) pointwise. Given $k \in \mathbb{N}$ and $\alpha \in(0,1)$, the small Hölder space $h^{k+\alpha}(\Sigma)$ is defined to be the completion of the smooth functions $C^{\infty}(\Sigma)$ in the $C^{k+\alpha}(\Sigma)$-norm. Also, given $U \subset \mathbb{R}^{3}$ open and bounded, the space $b u c^{k+\gamma}(U)$ stands for the closure of $B U C^{\infty}(U)$ in $B U C^{k+\alpha}(U)$. Combining the results of Lemmas 3.2, 3.3 and 3.4 shows that the solution of the problem (3.1) is determined if we can specify how the tumor evolves. More exactly, the concentration of nutrient in and outside the solid tumor, as well as the pressure, can be expressed in dependence of $\rho$, the concentration of nutrient on $\partial \Omega$ and the constants $A, B, C, D$. Therefore, we shall also call only $\rho$ as being solution of (3.1). The main result of this section is the following theorem.

Theorem 3.1 (Local well-posedness) There exists an open zero neighborhood $\mathcal{O} \subset h^{4+\alpha}(\Sigma) \cap$ Ad, such that for all $\rho_{0} \in \mathcal{O}$ problem (3.1) has a unique classical Hölder solution $\rho$ defined on a maximal time interval $t \in\left[0, T\left(\rho_{0}\right)\right)$, with $T\left(\rho_{0}\right)>0$, and which satisfies $\rho(t) \in \mathcal{O}$ for all $0 \leqslant t<T\left(\rho_{0}\right)$.

Although the new formulation (3.1) has the advantage that it transforms the problem of determining the evolution of the solid tumor into the problem of finding $\rho$, it has the inconvenience that the equations of (3.1) are posed on sets which depend on the unknown $\rho$ and change with time. That is why we transform (3.1) by writing all the equations of this system on the fixed reference manifolds $\Omega_{H}, \Omega_{T}$ and $\Sigma$. To do this, we extend $\theta_{\rho}$ to a diffeomorphism $\Theta_{\rho}$ of the whole space $\mathbb{R}^{3}$. For simplicity, we will write in the remaining part of the paper $\Theta_{\rho}$ for both diffeomorphisms. For this purpose, we choose a cut off function $\varphi \in C^{\infty}(\mathbb{R},[0,1])$ such that

$$
\varphi(\lambda)= \begin{cases}1 & \text { if } \quad|\lambda| \leqslant a / 4 \\ 0 & \text { if } \quad|\lambda| \geqslant 3 a / 4\end{cases}
$$

and the first derivative satisfies $\sup _{\mathbb{R}}\left|\varphi^{\prime}\right|<4 / a$. Then, given $\rho \in \operatorname{Ad}$, we define the extension $\Theta_{\rho}$ of $\theta_{\rho}$ by the relation

$$
\Theta_{\rho}(y):=\left\{\begin{array}{ll}
X(P(y), \Lambda(y)+\varphi(\Lambda(y)) \rho(P(y))) & \text { if } \quad y \in \mathscr{R} \\
y & \text { if } y \notin \mathscr{R}
\end{array} .\right.
$$

The condition imposed on $\varphi^{\prime}$ implies that for $|\rho|<a / 4$, the function $\lambda \mapsto \lambda+\varphi(\lambda) \rho$ is strictly increasing. Since $\Theta_{\rho}(y)=y$ for all $y \in \mathscr{R}$ with $|\Lambda(y)|>3 a / 4$, we conclude that $\Theta_{\rho}$ satisfies

$$
\Theta_{\rho} \in \operatorname{Diff}^{2}\left(\mathbb{R}^{3}\right) \cap \operatorname{Diff}^{2}(\Omega) \cap \operatorname{Diff}^{2}\left(\Omega_{T}, \Omega_{T}(\rho)\right) .
$$

The diffeomorphism $\Theta_{\rho}$ introduces pull-back and push-forward operators which associate to each function defined on the reference domains $\Omega_{T}$ and $\Omega_{H}:=\Omega_{H}(0)$ a unique function 
on $\Omega_{T}(\rho)$ and $\Omega_{H}(\rho)$, respectively, according to the following relations:

$$
\begin{aligned}
& \Theta_{\rho}^{*}: B U C(\Xi(\rho)) \rightarrow B U C(\Xi), u \mapsto u \circ \Theta_{\rho}, \\
& \Theta_{*}^{\rho}: B U C(\Xi) \rightarrow B U C(\Xi(\rho)), v \mapsto v \circ \Theta_{\rho}^{-1},
\end{aligned}
$$

with $\Xi$ being either $\Omega_{T}$ or $\Omega_{H}$. We use these operators to transform problem (3.1) into a problem on the fixed reference domains $\Omega_{T}$ and $\Omega_{H}$. To do this, we define the transformed operators $\mathscr{A}(\rho)$ and $\mathscr{B}(\rho)$ by the formulae

$$
\mathscr{A}(\rho) v:=\Theta_{\rho}^{*}\left(\Delta\left(\Theta_{*}^{\rho} v\right)\right), \quad \mathscr{B}(\rho) v:=\operatorname{tr}\left(\left\langle\nabla\left(\Theta_{*}^{\rho} v\right) \mid \nabla \mathscr{N}_{\rho}\right\rangle\left(\Theta_{\rho}\right)\right)
$$

for functions $\rho \in \operatorname{Ad}$ and $v \in b u c^{2+\alpha}(\Xi)$, with $\Xi$ as above. We write tr to denote the trace operator with respect to $\Sigma$. Lastly, we introduce the operator mapping functions $\rho \in \mathrm{Ad}$ onto the mean curvature of $\Sigma(\rho)$

$$
\mathscr{K}(\rho):=\Theta_{\rho}^{*} \kappa_{\Sigma(\rho)} .
$$

It is not difficult to see that if $\left(\rho, \sigma_{H}, \sigma_{T}, p\right)$ is a solution of (3.1), then the tuple $(\rho, v, u, q):=\left(\rho, \Theta_{\rho}^{*} \sigma_{H}, \Theta_{\rho}^{*} \sigma_{T}, \Theta_{\rho}^{*} p\right)$ solves pointwise a system of equations that are defined on the reference domains:

$$
\left\{\begin{aligned}
\mathscr{A}(\rho) u & =u & & \text { in } \Omega_{T}, \\
\mathscr{A}(\rho) v & =0 & & \text { in } \Omega_{H}, \\
\mathscr{A}(\rho) q & =A u+B & & \text { in } \Omega_{T}, \\
v & =1 & & \text { on } \Sigma, \\
u & =v & & \text { on } \Sigma, \\
q & =\mathscr{K}(\rho) & & \text { on } \Sigma, \\
D \mathscr{B}(\rho) v & =\mathscr{B}(\rho) u & & \text { on } \Sigma, \\
\partial_{t} \rho & =\mathscr{B}(\rho) q-C \mathscr{B}(\rho) u & & \text { on } \Sigma, \\
\rho(0) & =\rho_{0} & & \text { on } \Sigma,
\end{aligned}\right.
$$

with $t \in[0, T]$. The notion of solution for this problem is defined analogously to that of the solution to (3.1). In fact problems (3.1) and (3.5) are equivalent in the sense that the tuple $\left(\rho, \sigma_{H}, \sigma_{T}, p\right)$ is a solution of (3.1) if and only if $\left(\rho, \Theta_{\rho}^{*} \sigma_{H}, \Theta_{\rho}^{*} \sigma_{T}, \Theta_{\rho}^{*} p\right)$ is a classical solution of (3.5). This assertion is a direct consequence of the following lemma.

Lemma 3.2 Given $\rho \in h^{4+\alpha}(\Sigma) \cap \operatorname{Ad}$ and $\Xi \in\left\{\Omega_{T}, \Omega_{H}\right\}$, the operator

$$
\Theta_{\rho}^{*}: b u c^{2+\alpha}(\Xi(\rho)) \rightarrow b u c^{2+\alpha}(\Xi)
$$

is an isomorphism and $\left(\Theta_{\rho}^{*}\right)^{-1}=\Theta_{*}^{\rho}$.

Proof Pick $\rho \in \operatorname{Ad} \cap h^{4+\alpha}(\Sigma)$ and $\sigma_{T} \in b u c^{2+\alpha}\left(\Omega_{T}(\rho)\right)$. We show that $u:=\Theta_{\rho}^{*} \sigma_{T}$ belongs to $b u c^{2+\alpha}\left(\Omega_{T}\right)$. By the definition of the small Hölder spaces we may find sequences 
$\left(\rho_{n}\right)_{n} \subset C^{\infty}(\Sigma)$ and $\left(\sigma_{T n}\right) \subset B U C^{\infty}\left(\Omega_{T}(\rho)\right)$ with the property that $\rho_{n} \rightarrow \rho$ in $C^{4+\alpha}(\Sigma)$ and $\sigma_{T n} \rightarrow \sigma_{T}$ in $B U C^{2+\alpha}\left(\Omega_{T}(\rho)\right)$. We can also achieve that $\rho_{n}>\rho$, which ensures that $\Omega_{T}(\rho) \subset \Omega_{T}\left(\rho_{n}\right)$ provided $n$ is large enough to guarantee $\rho_{n} \in$ Ad. Consequently, we can define the composition $u_{n, m}:=\Theta_{\rho_{n}}^{*} \sigma_{T m}, n, m \in \mathbb{N}$, which is a smooth function, i.e. $u_{n, m} \subset B U C^{\infty}\left(\Omega_{T}\right)$. We then get:

$$
\begin{aligned}
u_{n, m}-u & =\sigma_{T m} \circ \Theta_{\rho_{n}}-\sigma_{T} \circ \Theta_{\rho} \\
& =\left(\sigma_{T m} \circ \Theta_{\rho_{n}}-\sigma_{T m} \circ \Theta_{\rho}\right)+\left(\sigma_{T m} \circ \Theta_{\rho}-\sigma_{T} \circ \Theta_{\rho}\right) \\
& =\left(\int_{0}^{1} \partial \sigma_{T m}\left((1-t) \Theta_{\rho_{n}}+t \Theta_{\rho}\right) d t\right)\left(\Theta_{\rho}-\Theta_{\rho_{n}}\right)+\left(\sigma_{T m}-\sigma_{T}\right) \circ \Theta_{\rho} .
\end{aligned}
$$

The last term can be made arbitrarily small by choosing $m$ large enough, while since

$$
\left\|\Theta_{\rho_{n}}-\Theta_{\rho}\right\|_{B U C^{4+\alpha}(\Omega)} \leqslant C\left\|\rho_{n}-\rho\right\|_{C^{4+\alpha}(\Sigma)}, \quad n \in \mathbb{N},
$$

we may choose $n$ sufficiently large to guarantee that the first term is also as small as we want. This shows that $\Theta_{\rho}^{*}: b u c^{2+\alpha}\left(\Omega_{T}(\rho)\right) \rightarrow b u c^{2+\alpha}\left(\Omega_{T}\right)$ is well defined. Using the same arguments, we get that $\Theta_{*}^{\rho}$ is also well defined and this proves our assertion when $\Xi=\Omega_{T}$. The case $\Xi=\Omega_{H}$ follows similarly.

We now proceed as in Section 2 and solve separately the diffraction problem for $(u, v)$ and afterwards the elliptic problem for the function $q$. By doing this we can express, since we now work on the fixed reference domains $\Omega_{H}$ and $\Omega_{T}$, the solutions of this problems as functions depending smoothly on the unknown $\rho$. Before doing this we study the regularity properties of the operators defined by (3.3) and (3.4). It was noticed in [17], that the operator $\mathscr{A}(\rho)$ is in fact the Laplace-Beltrami operator of the manifold $\left(\Omega, \Theta_{\rho}^{*} g\right)$,

$$
\mathscr{A}(\rho)=\Delta_{\Theta_{\rho}^{*} g}=\frac{1}{\sqrt{G(\rho)}} \sum_{i, j=1}^{3} \frac{\partial}{\partial x^{i}}\left(\sqrt{G(\rho)} g^{i j}(\rho) \frac{\partial}{\partial x^{j}}\right),
$$

where $\Theta_{\rho}^{*} g$ is the pull-back metric on $\Omega$ induced by the diffeomorphism $\Theta_{\rho}$ and the standard Euclidean metric $g$. We denoted by $g_{i j}(\rho):=\left\langle\partial_{i} \Theta_{\rho} \mid \partial_{j} \Theta_{\rho}\right\rangle, 1 \leqslant i, j \leqslant 3$ the coefficients of the first fundamental form, $G(\rho)$ is the determinant of $\left(g_{i j}(\rho)\right)$, and $\left(g^{i j}(\rho)\right)$ is its inverse. By definition, $\Theta_{\rho}(y)=P(y)+(\Lambda(y)+\varphi(\Lambda(y)) \rho(P(y))) v(P(y))$ for $y \in \mathscr{R}$ so that we have

$$
\left[\rho \mapsto \Theta_{\rho}\right] \in C^{\omega}\left(h^{4+\alpha}(\Sigma) \cap \operatorname{Ad}, b u c^{4+\alpha}\left(\Omega, \mathbb{R}^{3}\right)\right) .
$$

Consequently, we get that

$$
\mathscr{A} \in C^{\omega}\left(h^{4+\alpha}(\Sigma) \cap \operatorname{Ad}, \mathscr{L}\left(b u c^{2+\alpha}(\Xi), b u c^{\alpha}(\Xi)\right)\right) .
$$

Furthermore, by the chain rule we have

$$
\mathscr{B}(\rho) v=\operatorname{tr}\left(\left\langle\nabla\left(\Theta_{*}^{\rho} v\right) \mid \nabla \mathscr{N}_{\rho}\right\rangle\left(\Theta_{\rho}\right)\right)=\operatorname{tr}\left\langle\left[\partial \Theta_{\rho}^{-1}\left(\Theta_{\rho}\right)\right)\right]^{T} \nabla v\left|\nabla \mathscr{N}_{\rho}\left(\Theta_{\rho}\right)\right\rangle=\operatorname{tr}\langle b(\rho) \mid \nabla v\rangle
$$


for all $\rho \in h^{4+\alpha}(\Sigma) \cap$ Ad, and $v \in b u c^{2+\alpha}(\Xi)$, with $b(\rho):=\partial \Theta_{\rho}^{-1}\left(\Theta_{\rho}\right) \nabla \mathscr{N}_{\rho}\left(\Theta_{\rho}\right)$. Since

$$
\left[\rho \mapsto \mathscr{N}_{\rho}\right] \in C^{\omega}\left(\rho^{4+\alpha}(\Sigma) \cap \mathrm{Ad}, b u c c^{4+\alpha}(\mathscr{R})\right),
$$

and taking into account that $P\left(\Theta_{\rho}(y)\right)=P(y)$ for all $y \in \mathscr{R}$, we conclude that $\mathscr{B}$ is also analytic, i.e.

$$
\mathscr{B} \in C^{\omega}\left(h^{4+\alpha}(\Sigma) \cap \mathrm{Ad}, \mathscr{L}\left(b u c^{2+\alpha}(\Xi), h^{1+\alpha}(\Sigma)\right)\right) .
$$

These are the main ingredients used to prove the following lemma.

Lemma 3.3 Given $\rho \in h^{4+\alpha}(\Sigma) \cap \mathrm{Ad}$, there exists a unique solution

$$
\mathscr{T}(\rho)=\left(\mathscr{T}_{H}(\rho), \mathscr{T}_{T}(\rho)\right) \in b u c^{2+\alpha}\left(\Omega_{H}\right) \times b u c^{2+\alpha}\left(\Omega_{T}\right)
$$

of the transformed diffraction problem

$$
\left\{\begin{array}{rlrl}
\mathscr{A}(\rho) u & =u & & \text { in } \Omega_{T}, \\
\mathscr{A}(\rho) v & =0 & & \text { in } \Omega_{H}, \\
v & =1 & & \text { on } \Sigma, \\
u & =v & & \text { on } \Sigma, \\
D \mathscr{B}(\rho) v & =\mathscr{B}(\rho) u & \text { on } \Sigma .
\end{array}\right.
$$

Moreover, the function $\mathscr{T}$ is real analytic

$$
[\rho \mapsto \mathscr{T}(\rho)] \in C^{\omega}\left(h^{4+\alpha}(\Sigma) \cap \operatorname{Ad}, b u c^{2+\alpha}\left(\Omega_{H}\right) \times b u c^{2+\alpha}\left(\Omega_{T}\right)\right) .
$$

Proof Similar arguments as for (2.2) ensure that the system (3.9) has a unique classical solution $\mathscr{T}(\rho)$ in the classical Hölder spaces. To study the regularity of the solution operator, we define the Banach space

$$
\mathbb{E}:=\left\{(u, v) \in B U C^{2+\alpha}\left(\Omega_{H}\right) \times B U C^{2+\alpha}\left(\Omega_{T}\right): u=v \text { on } \Sigma\right\},
$$

and the function $\rho \mapsto \Psi(\rho):[(u, v) \mapsto(\mathscr{A}(\rho) u-u, \mathscr{A}(\rho) v, \operatorname{tr} v, D \mathscr{B}(\rho) v-\mathscr{B}(\rho) u)]$, which belongs, in view of (3.6) and (3.8), to

$$
\Psi \in C^{\omega}\left(C^{4+\alpha}(\Sigma) \cap \mathrm{Ad}, \mathscr{L}\left(\mathbb{E}, B U C^{\alpha}\left(\Omega_{H}\right) \times B U C^{\alpha}\left(\Omega_{T}\right) \times C^{2+\alpha}(\Sigma) \times C^{1+\alpha}(\Sigma)\right)\right) .
$$

Since $\Psi(\rho)$ is an isomorphism for all $\rho \in C^{4+\alpha}(\Sigma) \cap \operatorname{Ad}$ and $\mathscr{T}(\rho)=\Psi(\rho)^{-1}(0,0,1,0)$, we obtain by taking into account that the function mapping an isomorphism onto its inverse is analytic, that $\mathscr{T}(\rho)$ depends analytically on $\rho$. The assertion (3.10) follows now by using a density argument.

Before solving the Dirichlet problem for $q$, we study the dependence of the mean curvature operator $\mathscr{K}$ of $\rho$. Since $\Sigma(\rho)$ is the zero level set of $\mathscr{N}_{\rho}$, we can express the 
function $\mathscr{K}(\rho)$ by the formula

$$
\mathscr{K}(\rho)=\frac{1}{2} \operatorname{div}\left(\frac{\nabla \mathscr{N}_{\rho}}{\left|\nabla \mathscr{N}_{\rho}\right|}\right) \circ \Theta_{\rho},
$$

which yields together with (3.7)

$$
\mathscr{K} \in C^{\omega}\left(h^{4+\alpha}(\Sigma) \cap \mathrm{Ad}, h^{2+\alpha}(\Sigma)\right) .
$$

We close the preparations needed to prove Theorem 3.1 with the following lemma.

Lemma 3.4 Given $\rho \in h^{4+\alpha}(\Sigma) \cap \mathrm{Ad}$, the Dirichlet problem

$$
\left\{\begin{aligned}
\mathscr{A}(\rho) q & =A \mathscr{T}_{T}(\rho)+B & \text { in } & \Omega_{T}, \\
q & =\mathscr{K}(\rho) & \text { on } & \Sigma .
\end{aligned}\right.
$$

possesses a unique solution $\mathscr{S}(\rho) \in b_{u c}{ }^{2+\alpha}\left(\Omega_{T}\right)$. The function $\mathscr{S}$ is real analytic

$$
\mathscr{S} \in C^{\omega}\left(h^{4+\alpha}(\Sigma) \cap \operatorname{Ad}, b u c^{2+\alpha}\left(\Omega_{T}\right)\right) .
$$

Proof The proof is similar to that of [17, Lemma 3.2].

From Lemmas 3.3 and 3.4 we conclude that the tuple $(\rho, v, u, q)$ is a solution of (3.5) if and only if $(v(t), u(t))=\mathscr{T}(\rho(t)), q(t)=\mathscr{S}(\rho(t))$, and

$$
\partial_{t} \rho=\mathscr{B}(\rho) \mathscr{S}(\rho)-C \mathscr{B}(\rho) \mathscr{T}_{T}(\rho), \quad \rho(0)=\rho_{0} .
$$

This shows that the original problem (3.1) may be expressed as an abstract evolution equation for the function $\rho$, which describes the evolution of the solid tumor. We are now ready to prove our second main result.

Proof of Theorem 3.1 Let $\Phi: h^{4+\alpha}(\Sigma) \cap \operatorname{Ad} \rightarrow h^{1+\alpha}(\Sigma)$ be the nonlinear and non-local operator with $\Phi(\rho):=\mathscr{B}(\rho) \mathscr{S}(\rho)-C \mathscr{B}(\rho) \mathscr{T}_{T}(\rho)$. Invoking (3.10) and (3.13) we obtain that $\Phi$ is a real analytic mapping, i.e. $\Phi \in C^{\omega}\left(h^{4+\alpha}(\Sigma) \cap \mathrm{Ad}, h^{1+\alpha}(\Sigma)\right)$, and system (3.1) is equivalent to the abstract Cauchy problem

$$
\partial_{t} \rho=\Phi(\rho), \quad \rho(0)=\rho_{0} .
$$

The key point in the proof is to show that the Fréchet derivative $\partial \Phi(0)$ generates an analytic semi-group. To prove this assertion, we proceed as in [12] and decompose $\partial \Phi(0)=\mathfrak{N} \circ \partial \mathscr{K}(0)+P_{1}$, where $P_{1}$ is a linear operator having first order, and $\mathfrak{N}$ is the Dirichlet-Neumann operator, which associates to each function in $\rho \in h^{2+\alpha}(\Sigma)$ the outward normal derivative of the harmonic function in $\Omega_{T}$ equal to $\rho$ on $\Sigma$, i.e.

$$
\left[\rho \mapsto \mathfrak{N}(\rho):=\partial_{v}(\Delta, \operatorname{tr})^{-1}(\rho)\right] \in \mathscr{L}\left(h^{2+\alpha}(\Sigma), h^{1+\alpha}(\Sigma)\right)
$$


Using local charts, it is shown in [17, Remark 3.3] that in fact $\partial \mathscr{K}(0)=\Delta_{\pi}^{\Sigma}+P_{2}$, where $\Delta_{\pi}^{\Sigma}$ is the principal part of the Laplace-Beltrami operator on $(\Sigma, \eta), \eta$ being the metric induced on $\Sigma$ by the Euclidean metric $g$ in $\mathbb{R}^{3}$. Hence, $\partial \Phi(0)=\mathfrak{N} \Delta_{\pi}^{\Sigma}+P$, with $\mathfrak{N} \Delta_{\pi}^{\Sigma}$ being an operator of third order and $P:=P_{1}+\mathfrak{N} P_{2}$ having lower order. We infer from [16, Theorem 6.12] by using standard perturbation arguments that $\partial \Phi(0)$ generates a strongly continuous analytic semigroup, i.e.

$$
-\partial \Phi(0) \in \mathscr{H}\left(h^{4+\alpha}(\Sigma), h^{1+\alpha}(\Sigma)\right) .
$$

The assertion of Theorem 3.1 is obtained by using [26, Theorem 8.4.1] as we did in the proof of [12, Theorem 2.1].

\section{Stability properties of the radially symmetric equilibria}

The main goal of this section is to study the stability properties of the radially symmetric steady states determined in Theorem 2.1. The analysis done in the previous sections is very useful because we have reduced the original problem (1.2) to an abstract nonlinear Cauchy problem which suits the application of the principle of linearized stability. To be more precise, pick $R_{1} \in\left(R_{1 *}, R_{1}^{*}\right)$ and let $\Omega:=\mathbb{B}\left(0, R_{2}\right)$, where $R_{2}$, given by (2.9), be the fixed region where the tumor is observed. At rest, the solid tumor is located at $\mathbb{B}\left(0, R_{1}\right)$, which corresponds to the stationary solution $\rho_{*} \equiv 0$ of (3.1) if we choose in addition $\Sigma:=R_{1} \mathbb{S}^{2}$. The question that we arise is, what happens with a tumor which is initially close to $\Sigma$. This is equivalent to studying the stability properties of the steady state $\rho_{*} \equiv 0$ of problem (3.1). We shall prove that as follows.

Theorem 4.1 (Unstable tumor growth) Let $A \in \mathbb{R}$ and let $B, C, D$ be positive constants satisfying (2.11). There exist constants $\varpi>0$ and $R_{1}^{*} \in\left(R_{1^{*}}, R_{1}^{*}\right)$ depending only on $A, B, C$ such that if $D<1+\varpi$ and $R_{1} \in\left(R_{1}^{*}, R_{1}^{*}\right)$, then the equilibrium $\rho_{*} \equiv 0$ of (3.1) is unstable.

Remark 4.2 Invoking Remark 2.2, the condition that $R_{1}$ is close to $R_{1}^{*}$ implies that $R_{2}$, the radius of $\Omega$, is nearby $R_{1}$, meaning that the solid tumor is relatively close to the source of nutrient. Moreover, in this unstable growth regime $D_{H}<(1+\bar{\omega}) D_{T}$, meaning that the nutrient may diffuse more rapidly through the solid tumor than in the surrounding tissue.

Numerical simulation done in [3] shows that tumors being initially circular may drive fingering instabilities. Theorem 4.1 states that this may well be the case also when starting close to a stationary tumor.

We now prove that if the $D,(C-A) / B$ and $C /(C-A)$ are sufficiently large, then the solid tumor attracts at an exponential rate all solutions of (3.1) that start nearby. Particularly, if for some sufficiently large integer $N \in \mathbb{N}$, we have

$$
\frac{D_{H}}{D_{T}} \geqslant N, \quad \frac{\lambda_{p} \sigma_{\infty}}{\lambda_{A}} \geqslant N, \quad \text { and } \frac{\chi_{\sigma} \lambda_{\chi}}{\lambda_{p} \sigma_{\infty}} \geqslant N,
$$


cf. (1.1) and (1.4), then the radially symmetric steady states are exponentially stable. This shows that the taxis and the nutrient uptake rate also influence the stability properties of the equilibria.

Theorem 4.3 (Asymptotically stable steady states) Let $A \in \mathbb{R}$ and $B, C, D$ be positive constants satisfying (2.11). In addition, we assume that:

$$
D>1+\frac{6}{\operatorname{coth}(1)-1}
$$

Denoting by $R_{\odot}$ the unique solution of the equation

$$
R^{2}+\frac{R^{2} \sinh (R)}{R \cosh (R)-\sinh (R)}=\frac{3(C-A)}{B},
$$

we presuppose that

$$
R_{\odot} \geqslant 1 \quad \text { and } \quad \frac{1}{D} \geqslant \frac{\sinh \left(R_{\odot}\right)}{R_{\odot} \cosh \left(R_{\odot}\right)-\sinh \left(R_{\odot}\right)} .
$$

Finally, assume that

$$
\frac{C}{C-A} \geqslant 3 \sup _{\left(k, R_{1}\right)}\left|\frac{R_{1} \sinh \left(R_{1}\right)}{3 Q}-\frac{1}{R_{1}}\right| \frac{(2 k+1) D \sinh \left(R_{1}\right)+R_{1} \cosh \left(R_{1}\right)-\sinh \left(R_{1}\right)}{(D-1)\left(k^{2}+k\right)},
$$

where the supremum is taken over all pairs $\left(k, R_{1}\right) \in \mathbb{N} \times\left(R_{\odot}, R_{1}^{*}\right)$ and $R_{1}^{*}$ is given by (2.12). Then for all $R_{1} \in\left(R_{1^{*}}, R_{1}^{*}\right)$, the stationary solution $\rho_{*} \equiv 0$ of (3.1) is exponentially stable. More precisely, there exist positive constants $\delta, M, w$ such that for all $\rho \in h^{4+\alpha}(\Sigma)$ with $\|\rho\|_{h^{4+\alpha}(\Sigma)}<\delta$, the solution to (3.1) exists globally and

$$
\|\rho(t)\|_{h^{4+\alpha}(\Sigma)}+\left\|\partial_{t} \rho(t)\right\|_{h^{1+\alpha}(\Sigma)} \leqslant M e^{-w t}\left\|\rho_{0}\right\|_{h^{4+\alpha}(\Sigma)} \quad \text { for all } t \geqslant 0 .
$$

Remark 4.4 We now show that conditions (4.1)-(4.4) are satisfied for a large range of values for the parameters $(A, B, C, D)$. From the discussion in Section 2, we know that the left-hand side of (4.2) is strictly increasing in $R$. Moreover, the function $h(R):=$ $\sinh (R) /(R \cosh (R)-\sinh (R))$ is strictly decreasing to 0 as $R \rightarrow \infty$. Hence, if $(C-A) / B$ is large enough, the solution of (4.2) will satisfy (4.3). A consequence of (2.12) and (4.1) is that $R_{1^{*}}>R_{\odot}$, and if we choose $(C-A)$ as small enough, this guarantees (4.4). We enhance that our choice (4.1)-(4.4) for $(A, B, C, D)$ are not optimal and could be slightly improved.

The theorem states that equilibria are exponentially stable if the relative rate of mitosis to taxis is small, the relative rate of mitosis to apoptosis is large and the diffusion constant is large enough.

The size of the value $D=D_{H} / D_{T}$ is essential for the stability properties of the steady states: If $D$ is below or very close to 1 , the steady states are unstable, whereas if $D_{T} \ll D_{H}$, the equilibria are stable. Our analysis however gives no evidence upon stability properties of steady states for values of $D$ which are not too large but exceed the threshold $1+\bar{\omega}$ 
(where $\bar{\omega}$ appears in Theorem 4.1). Particularly, Theorem 4.1 shows that in a physically relevant situation when the diffusion coefficients in the surrounding tissue and the tumor are almost equal, the tumors exhibit an unstable growth. On the other hand, in the absence of taxis the diffusion constant outside the tumor is large, cf. [3], and the stability properties of the equilibria are predicted by Theorem 4.3.

In order to prove these results we have to study the spectrum of the the Fréchet derivative $\partial \Phi(0)$. Invoking that $\partial \Phi(0)$ is a generator of an analytic semi-group and taking also into account that the embedding $h^{4+\alpha}(\Sigma) \hookrightarrow h^{1+\alpha}(\Sigma)$ is compact, we obtain that $\partial \Phi(0)$ has compact resolvent. Consequently, the operator $\partial \Phi(0)$ has only point spectrum, i.e.

$$
\sigma(\partial \Phi(0))=\{\lambda \in \mathbb{C}: \lambda \text { is eigenvalue of } \partial \Phi(0)\} .
$$

We show below that the operator $\partial \Phi(0)$ is the Fourier multiplication operator acting on orthonormal basis given by spherical harmonics. Then the point spectrum of $\partial \Phi(0)$ is exactly the symbol of this multiplier, so that we are finally left to decide about the sign of this symbol.

We now proceed and determine the linearization of problem (3.1) at the radially symmetric solution $\left(\rho_{*} \equiv 0, \sigma_{H}, \sigma_{T}, p\right)$ given by (2.3)-(2.5) and (2.7). Therefore, we consider linear perturbations of this solution of the form

$$
\begin{array}{ll}
\rho_{\varepsilon}(\omega):=\varepsilon \rho(\omega), & \sigma_{H}^{\varepsilon}(y)=\sigma_{H}(r)+\varepsilon v(r, \omega), \\
\sigma_{T}^{\varepsilon}(y)=\sigma_{T}(r)+\varepsilon u(r, \omega), & p^{\varepsilon}(y)=p(r)+\varepsilon q(r, \omega),
\end{array}
$$

where $r=|y|, \omega=y /|y|, \varepsilon$ is a small parameter and $(\rho, v, u, q)$ are unknown functions. We have identified functions of $\Sigma$ with functions on $\mathbb{S}^{2}$, and $(v, u, q)$ are defined on the perturbed domains

$$
\Omega_{T}\left(\rho_{\varepsilon}\right)=\left\{(r, \omega): r<R_{1}+\varepsilon \rho(\omega)\right\}, \quad \Omega_{H}\left(\rho_{\varepsilon}\right)=\left\{(r, \omega): R_{1}+\varepsilon \rho(\omega)<r<R_{2}\right\} .
$$

Letting $\Delta_{\omega}$ be the Laplace-Beltrami operator on the unit sphere $\mathbb{S}^{2}$, we easily see from (2.2) that the linearizations of the first three equations of (3.1) are

$$
\begin{aligned}
& \frac{\partial^{2} u}{\partial r^{2}}+\frac{2}{r} \frac{\partial u}{\partial r}+\frac{1}{r^{2}} \Delta_{\omega} u=u \quad \text { in } \quad \Omega_{T}, \\
& \frac{\partial^{2} v}{\partial r^{2}}+\frac{2}{r} \frac{\partial v}{\partial r}+\frac{1}{r^{2}} \Delta_{\omega} v=0 \quad \text { in } \quad \Omega_{H}, \\
& \frac{\partial^{2} q}{\partial r^{2}}+\frac{2}{r} \frac{\partial q}{\partial r}+\frac{1}{r^{2}} \Delta_{\omega} q=A u \quad \text { in } \quad \Omega_{T},
\end{aligned}
$$

respectively. Besides, it is also immediate to see that the linearizations of the first two boundary conditions of (3.1) are given by

$$
\begin{gathered}
v=0 \quad \text { on } \partial \Omega, \\
v-u=(D-1) \sigma_{H}^{\prime}\left(R_{1}\right) \eta \quad \text { on } \Sigma .
\end{gathered}
$$


Note that in this particular situation there is an explicit formula for the function $\mathscr{N}_{\rho}$ defined in Section 2. Namely, we have $\mathscr{N}_{\rho}(y)=|y|-R_{1}-\rho(\omega)$ for $y \in \mathscr{R},(\mathscr{R}$ is an annular domain in this case), which leads us in our case to the following expression for the outward normal at $\partial \Omega_{T}\left(\rho_{\varepsilon}\right)$

$$
v_{\varepsilon}=\frac{\omega-\varepsilon \nabla_{\omega} \rho(\omega)}{\left|\omega-\varepsilon \nabla_{\omega} \rho(\omega)\right|}=\omega-\varepsilon \nabla_{\omega} \rho(\omega)+o(\varepsilon),
$$

where $\nabla_{\omega}$ denotes the tangent mapping on the sphere $\mathbb{S}^{2}$, or the orthogonal projection of the gradient $\nabla$ to the tangent space $T_{\omega}\left(\mathbb{S}^{2}\right)$, when regarding a function in $\omega \in \mathbb{S}^{2}$ as a function in $y \in \mathbb{R}^{3}$ so that $\left\langle\omega \mid \nabla_{\omega}\right\rangle=0$. Since

$$
\begin{aligned}
\frac{\left\langle\nabla \sigma_{T}^{\varepsilon} \mid v_{\varepsilon}\right\rangle\left(\Theta_{\rho_{\varepsilon}}\right)-\left\langle\nabla \sigma_{T} \mid v\right\rangle\left(\Theta_{0}\right)}{\varepsilon} & =\frac{\left\langle\nabla \sigma_{T} \mid v_{\varepsilon}\right\rangle\left(\Theta_{\rho_{\varepsilon}}\right)+\varepsilon\left\langle\nabla u \mid v_{\varepsilon}\right\rangle\left(\Theta_{\rho_{\varepsilon}}\right)-\left\langle\nabla \sigma_{T} \mid v\right\rangle\left(\Theta_{0}\right)}{\varepsilon} \\
& =\left\langle\nabla u \mid v_{\varepsilon}\right\rangle\left(\Theta_{\rho_{\varepsilon}}\right)+\frac{\left\langle\nabla \sigma_{T} \mid v_{\varepsilon}\right\rangle\left(\Theta_{\rho_{\varepsilon}}\right)-\left\langle\nabla \sigma_{T} \mid v\right\rangle\left(\Theta_{0}\right)}{\varepsilon}
\end{aligned}
$$

we obtain, by using the relation $\nabla w=(\partial w / \partial r) \omega$, which holds true for radial symmetric functions $w$ and in particular for $w=\sigma_{T}$, that

$$
\lim _{\varepsilon \rightarrow 0} \frac{\left\langle\nabla \sigma_{T}^{\varepsilon} \mid v_{\varepsilon}\right\rangle\left(\Theta_{\rho_{\varepsilon}}\right)-\left\langle\nabla \sigma_{T} \mid v\right\rangle\left(\Theta_{0}\right)}{\varepsilon}=\partial_{\nu} u+\sigma_{T}^{\prime \prime}\left(R_{1}\right) \rho .
$$

Similar relations are also satisfied by $\sigma_{H}^{\varepsilon}$ and $p^{\varepsilon}$, with the obvious modifications. Therewith, the linearization of the sixth equation of (3.1) takes the following form,

$$
D \partial_{v} v-\partial_{v} u=\left(\sigma_{T}^{\prime \prime}\left(R_{1}\right)-D \sigma_{H}^{\prime \prime}\left(R_{1}\right)\right) \rho \quad \text { on } \quad \Sigma,
$$

which, in view of (2.2), is equivalent to

$$
D \partial_{v} v-\partial_{v} u=\sigma_{T}\left(R_{1}\right) \rho \quad \text { on } \quad \Sigma .
$$

Similarly, the linearization of the last equation of (3.1) is given by

$$
\partial_{t} \rho=-\partial_{\nu} q+C \partial_{v} u+\left((C-A) \sigma_{T}\left(R_{1}\right)-B\right) \rho \quad \text { on } \quad \Sigma .
$$

Finally, we have the following formula for the mean curvature of the hypersurface $\Sigma(\varepsilon \rho(\omega))$, cf. [7,21],

$$
\mathscr{K}\left(\rho_{\varepsilon}\right)=\frac{1}{R_{1}}-\frac{\varepsilon}{R_{1}^{2}}\left[\rho+\frac{1}{2} \Delta_{\omega} \rho\right]+o(\varepsilon),
$$

and we conclude that the linearization of the seventh equation in (3.1) reads as follows:

$$
q=-\frac{1}{R_{1}^{2}}\left[\rho+\frac{1}{2} \Delta_{\omega} \rho\right] \quad \text { on } \quad \Sigma .
$$

Summarizing, we have proved the following lemma.

Lemma 4.5 Let $R_{1} \in\left(R_{1^{*}}, R_{1}^{*}\right), R_{2}$ be given by (2.9), $\Sigma=R_{1} \mathbb{S}^{2}$ and $\Omega=\mathbb{B}\left(0, R_{2}\right)$. Then, the linearization of the problem (3.1) at the radially symmetric stationary solution $\left(\rho_{*} \equiv\right.$ $\left.0, \sigma_{H}, \sigma_{T}, p\right)$ found in Section 2 is given by the problem (4.5)-(4.12). 
When studying the linearized system (4.5)-(4.12), we proceed as we did in Section 3. Namely, given $\rho \in h^{4+\alpha}(\Sigma)$, we can solve the diffraction problem (4.5), (4.6) and (4.8)(4.10) for $(v, u)$ and obtain, similarly as we did in Lemma 3.3, an operator $(u, v)=(u, v)(\rho)$ expressing the solution of this problem in dependence of $\rho$ only. We now plug $u(\rho)$ into (4.7) and determine the solution $q=q(\rho)$ for the Dirichlet problem (4.7) and (4.12). These arguments show that the linearized problem (4.5)-(4.12) is equivalent to a linear evolution equation

$$
\partial_{t} \rho=\Phi_{L}(\rho), \quad t \geqslant 0,
$$

where $\Phi_{L}: h^{4+\alpha}(\Sigma) \rightarrow h^{1+\alpha}(\Sigma)$ is defined by the relation

$$
\Phi_{L}(\rho):=-\partial_{v}(q(\rho))+C \partial_{v}(u(\rho))+\left((C-A) \sigma_{T}\left(R_{1}\right)-B\right) \rho .
$$

Since problem (3.1) is equivalent to the transformed problem (3.5), we conclude from Lemma 4.5 that the Fréchet derivatives $\partial \Phi(0)$ and $\Phi_{L}$ coincide, i.e.

$$
\partial \Phi(0)[\rho]=\Phi_{L}(\rho) \quad \text { for all } \rho \in h^{4+\alpha}(\Sigma) \text {. }
$$

Since $\Sigma=R_{1} \mathbb{S}^{2}$, we will solve the linearized problem and find for each $\rho \in h^{4+\alpha}(\Sigma)$ an explicit representation formula for $\Phi_{L}(\rho)$. Therefore, given $k \geqslant 0$, we let $Y_{k l}, l=1, \ldots, d_{k}$, denote the spherical harmonics of degree $k$. Since they build an orthonormal basis in $L^{2}\left(\mathbb{S}^{2}\right)$, we expand $(\rho, v, u, q)$ in the following way:

$$
\begin{aligned}
& \rho=\sum_{k=0}^{\infty} \sum_{l=1}^{d_{k}} c_{k l} Y_{k l}(\omega) \quad v=\sum_{k=0}^{\infty} \sum_{l=1}^{d_{k}} v_{k l}(r) Y_{k l}(\omega), \\
& u=\sum_{k=0}^{\infty} \sum_{l=1}^{d_{k}} u_{k l}(r) Y_{k l}(\omega), q=\sum_{k=0}^{\infty} \sum_{l=1}^{d_{k}} q_{k l}(r) Y_{k l}(\omega),
\end{aligned}
$$

where $c_{k l}$ is assumed to be given, and $v_{k l}, u_{k l}$ and $p_{k l}$ are unknown.

Substituting (4.14) into the linearized problem (4.5)-(4.12), using the relation

$$
\Delta_{\omega} Y_{k l}=-\lambda_{k} Y_{k l}, \quad \lambda_{k}=k^{2}+k, \quad k \geqslant 1,
$$

and comparing coefficients of each $Y_{k l}$, we obtain a system of ordinary differential equations for the coefficients $\left(v_{k l}, u_{k l}\right), k \geqslant 0,1 \leqslant l \leqslant d_{k}$ :

$$
\left\{\begin{array}{l}
u_{k l}^{\prime \prime}+\frac{2}{r} u_{k l}^{\prime}-\frac{\lambda_{k}}{r^{2}} u_{k l}=u_{k l}, \quad 0<r<R_{1}, \\
v_{k l}^{\prime \prime}+\frac{2}{r} v_{k l}^{\prime}-\frac{\lambda_{k}}{r^{2}} v_{k l}=0, \quad R_{1}<r<R_{2}, \\
v_{k l}\left(R_{2}\right)=0, \\
v_{k l}\left(R_{1}\right)-u_{k l}\left(R_{1}\right)=(D-1) \sigma_{H}^{\prime}\left(R_{1}\right) c_{k l}, \\
D v_{k l}^{\prime}\left(R_{1}\right)-u_{k l}^{\prime}\left(R_{1}\right)=\sigma_{T}\left(R_{1}\right) c_{k l} .
\end{array}\right.
$$


Similarly, the coefficients $q_{k l}$ are the solutions of the following problem:

$$
\left\{\begin{array}{l}
q_{k l}^{\prime \prime}+\frac{2}{r} q_{k l}^{\prime}-\frac{\lambda_{k}}{r^{2}} q_{k l}=A u_{k l}, \quad 0<r<R_{1}, \\
q_{k l}\left(R_{1}\right)=\frac{\lambda_{k}-2}{2 R_{1}^{2}} c_{k l},
\end{array}\right.
$$

while the operator $\Phi_{L}$ is given by

$$
\sum_{k=0}^{\infty} \sum_{l=1}^{d_{k}} c_{k l} Y_{k l} \stackrel{\Phi_{L}}{\longmapsto} \sum_{k=0}^{\infty} \sum_{l=1}^{d_{k}}\left\{-q_{k l}^{\prime}\left(R_{1}\right)+C u_{k l}^{\prime}\left(R_{1}\right)+\left[(C-A) \sigma_{T}\left(R_{1}\right)-B\right] c_{k l}\right\} Y_{k l} .
$$

We now proceed and solve the systems (4.15) and (4.16) one by one. Note from (4.17) that in order to determine the function $\Phi_{L}(\rho)$ it suffices to find only the derivatives $-q_{k l}^{\prime}\left(R_{1}\right)$ and $u_{k l}^{\prime}\left(R_{1}\right)$. As in $[6,12]$, we let for each $n \in \mathbb{N}, \bar{u}_{n}$ denote the solution of the linear initial value problem

$$
\left\{\begin{array}{l}
\bar{u}_{n}^{\prime \prime}+\frac{2 n+2}{r} \bar{u}_{n}^{\prime}=\bar{u}_{n}, \quad r>0, \\
\bar{u}_{n}(0)=1, \bar{u}_{n}^{\prime}(0)=0 .
\end{array}\right.
$$

It is shown in [6] that the solution $\bar{u}_{n}$ is global and smooth, i.e. $u_{n} \in C^{\infty}([0, \infty))$. With this notation we have

$$
u_{k l}(r)=\alpha_{k l} r^{k} \bar{u}_{k}(r)
$$

for all $0 \leqslant r \leqslant R_{1}$, whereby the constants $\alpha_{k l}$ are still to be determined. The general solution of the second equation of (4.15) has the form

$$
v_{k l}(r)=\beta_{k l} r^{k}+\gamma_{k l} r^{-k-1},
$$

and the third relation of (4.15) implies that $\gamma_{k l}=-\beta_{k l} R_{2}^{2 k+1}$. From the last two equations of the system we finally get $\alpha_{k l}=: s_{k} c_{k l}$, where

$$
s_{k}=-\frac{\sigma_{T}\left(R_{1}\right)\left(R_{1} R_{2}^{2 k+1}-R_{1}^{2 k+2}\right)+D(D-1) \sigma_{H}^{\prime}\left(R_{1}\right)\left(k R_{1}^{2 k+1}+(k+1) R_{2}^{2 k+1}\right)}{\bar{u}_{k}\left(R_{1}\right)\left((D-1) k R_{1}^{3 k+1}+((k+1) D+k) R_{1}^{k} R_{2}^{2 k+1}\right)+R_{1}^{k+1} \bar{u}_{k}^{\prime}\left(R_{1}\right)\left(R_{2}^{2 k+1}-R_{1}^{2 k+1}\right)} .
$$

The denominator is positive because the functions $\bar{u}_{n}$ are strictly increasing, cf. [6,12]. Now that we know $u_{k l}$, we can pursue and solve the problem for $q_{k l}$. One can easily check that the solution of (4.16) is given by the relation

$$
q_{k l}(r)=A \alpha_{k l} r^{k} \bar{u}_{k}(r)+\delta_{k l} r^{k},
$$

where $\alpha_{k l}$ are given by (4.19) and the constant $\delta_{k l}$ can be determined from the last equation of (4.16)

$$
\delta_{k l}:=\frac{\lambda_{k}-2}{2 R_{1}^{k+2}} c_{k l}-A \alpha_{k l} \bar{u}_{k}\left(R_{1}\right) .
$$

Summarizing, we get from (4.17) that the Fréchet derivative of $\partial \Phi(0)$ is a Fourier multiplier

$$
\partial \Phi(0) \sum_{k=0}^{\infty} \sum_{l=1}^{d_{k}} c_{k l} Y_{k l}=\sum_{k=0}^{\infty} \sum_{l=1}^{d_{k}} S_{k} c_{k l} Y_{k l}
$$


where the symbol $S_{k}$ is given by

$$
S_{k}:=\frac{k\left(2-\lambda_{k}\right)}{2 R_{1}^{3}}+(C-A) R_{1}^{k} \bar{u}_{k}^{\prime}\left(R_{1}\right) s_{k}+C k R_{1}^{k-1} \bar{u}_{k}\left(R_{1}\right) s_{k}+(C-A) \sigma_{T}\left(R_{1}\right)-B
$$

for $k \in \mathbb{N}$. It is easy to read from (4.20) that the point spectrum of $\partial \Phi(0)$ consists exactly the real numbers $S_{k}$ so that in conclusion $\sigma(\partial \Phi(0))=\left\{S_{k}: k \in \mathbb{N}\right\}$. The maximum principle argument used in [12] shows that

$$
\bar{u}_{n+1} \leqslant \bar{u}_{n}, \quad \bar{u}_{n+1}^{\prime} \leqslant \bar{u}_{n}^{\prime}, \quad \bar{u}_{n}\left(R_{1}\right) \searrow 1, \bar{u}_{n}^{\prime}\left(R_{1}\right) \searrow 0,
$$

while for $n=0$ we have already shown in Section 2 that

$$
\bar{u}_{0}(r)=\frac{\sinh (r)}{r} \quad \text { for all } r \geqslant 0
$$

If we combine all these relations we obtain that

$$
\lim _{k \rightarrow \infty} S_{k}=-\infty
$$

This behavior was also suggested by the fact that $\partial \Phi(0)$ is a generator of an analytic semi-group. We now proceed with the proof of Theorem 4.1.

Proof of Theorem 4.1 In view of (4.24) it suffices to prove that if the hypotheses of Theorem 4.1 are fulfilled, then $S_{0}$ is positive. To do this, we choose $k=0$ in (4.19) and obtain the following relation

$$
s_{0}=-\frac{\sigma_{T}\left(R_{1}\right) R_{1}^{2} \frac{R_{2}-R_{1}}{R_{2}}+D(D-1) R_{1} \sigma_{H}^{\prime}\left(R_{1}\right)}{D \sinh \left(R_{1}\right)+\frac{R_{2}-R_{1}}{R_{2}} Q},
$$

where we used the shorthand $Q:=R_{1} \cosh \left(R_{1}\right)-\sinh \left(R_{1}\right)$. We rewrite this expression by using the results from Section 2. Invoking (2.3), (2.4) and (2.9), we compute that

$$
\sigma_{T}\left(R_{1}\right)=\frac{D \sinh \left(R_{1}\right)}{D \sinh \left(R_{1}\right)+\frac{R_{2}-R_{1}}{R_{2}} Q} \quad \text { and } \quad \sigma_{H}^{\prime}\left(R_{1}\right)=\frac{R_{1}^{-1} Q}{D \sinh \left(R_{1}\right)+\frac{R_{2}-R_{1}}{R_{2}} Q}
$$

where

$$
\frac{R_{2}-R_{1}}{R_{2}}=\frac{3(C-A) D Q-B D R_{1}^{2} \sinh \left(R_{1}\right)}{B R_{1}^{2} Q},
$$

so that we finally obtain an expression for $\sigma_{T}\left(R_{1}\right), \sigma_{H}^{\prime}\left(R_{1}\right)$ in dependence only of $R_{1}$ and the constants $A, B, C$ and $D$ :

$$
\sigma_{T}\left(R_{1}\right)=\frac{B R_{1}^{2} \sinh \left(R_{1}\right)}{3(C-A) Q} \quad \text { and } \quad \sigma_{H}^{\prime}\left(R_{1}\right)=\frac{B R_{1}}{3(C-A) D} .
$$


We conclude that

$$
s_{0}=-\frac{B R_{1}^{4} \sinh \left(R_{1}\right)}{9(C-A)^{2} Q^{3}}\left[3(C-A) Q-B R_{1}^{2} \sinh \left(R_{1}\right)\right]+\frac{1-D}{D} \frac{B^{2} R_{1}^{4}}{9(C-A)^{2} Q} .
$$

Note that $S_{0}$ is positive if and only if

$$
(C-A) \sigma_{T}\left(R_{1}\right)>B-(C-A) R_{1} \bar{u}_{0}^{\prime}\left(R_{1}\right) s_{0}=B-(C-A) \frac{Q}{R_{1}} s_{0} .
$$

We reformulate this condition by making use of the relations found above, and finally get that $S_{0}$ is positive exactly when

$$
\frac{R_{1}^{2} \sinh \left(R_{1}\right)}{3 Q}>1+\frac{B R_{1}^{3} \sinh \left(R_{1}\right)}{3(C-A) Q}\left[\frac{(C-A)}{B}-\frac{R_{1}^{2} \sinh \left(R_{1}\right)}{3 Q}\right]-\frac{1-D}{D} \frac{B R_{1}^{3}}{9(C-A)} .
$$

If we let $R_{1} \rightarrow R_{1}^{*}$ in (4.25), we obtain, in view of (2.12), the following relation:

$$
\frac{C-A}{B} \geqslant 1-\frac{1-D}{D} \frac{B R_{1}^{* 3}}{9(C-A)} \text {. }
$$

The assertion of the theorem follows now from the principle of linearized stability [26, Theorem 9.1.3] and (4.24).

We end this paper with the proof of our last result.

Proof of Theorem 4.3 Let $A, B, C$ and $D$ satisfy the assumptions of Theorem 4.3 and pick $R_{1} \in\left(R_{1 *}, R_{1}^{*}\right)$. Particularly, we have $D>1$. We claim that the spectrum $\sigma(\partial \Phi(0))$ is bounded away from zero in the negative half-plane. To do this, we show in a first step that $S_{0}<0$, and then that all $S_{k}, k \geqslant 1$, are negative.

In view of (4.25), $S_{0}$ is negative if and only if

$$
\frac{R_{1}^{2} \sinh \left(R_{1}\right)}{3 Q}<1+\frac{B R_{1}^{3} \sinh \left(R_{1}\right)}{3(C-A) Q}\left[\frac{(C-A)}{B}-\frac{R_{1}^{2} \sinh \left(R_{1}\right)}{3 Q}\right]-\frac{1-D}{D} \frac{B R_{1}^{3}}{9(C-A)}
$$

Hence, it suffices to prove that

$$
\frac{R_{1}^{2} \sinh \left(R_{1}\right)}{3 Q} \leqslant \frac{D-1}{D} \frac{B R_{1}^{3}}{9(C-A)} .
$$

We divide the relation by $R_{1}$ and obtain

$$
\frac{R_{1} \sinh \left(R_{1}\right)}{3 Q} \leqslant \frac{D-1}{D} \frac{B R_{1}^{2}}{9(C-A)} .
$$

The function $x \mapsto x \sinh (x) /(x \cosh (x)-\sinh (x))$ is strictly decreasing on $(0, \infty), R_{1}>$ $R_{1 *}>R_{\odot}$ (see Remark 4.4), and from (4.3) we also have $R_{\odot} \geqslant 1$. Consequently, we can bound the left-hand side of (4.26) by

$$
\frac{R_{1} \sinh \left(R_{1}\right)}{3 Q}<\frac{1}{\operatorname{coth}(1)-1} .
$$


On the other hand, we infer from (2.12) that

$$
\frac{R_{1}^{2}}{D}+\frac{R_{1}^{2} \sinh \left(R_{1}\right)}{R_{1} \cosh \left(R_{1}\right)-\sinh \left(R_{1}\right)} \geqslant \frac{R_{1 *}^{2}}{D}+\frac{R_{1^{*}}^{2} \sinh \left(R_{1 *}^{2}\right)}{R_{1^{*}}^{2} \cosh \left(R_{1 *}^{2}\right)-\sinh \left(R_{1^{*}}^{2}\right)}=\frac{3(C-A)}{B},
$$

and obtain after dividing the inequality by $R_{1}^{2}$ the following relation:

$$
\frac{1}{D}+\frac{\sinh \left(R_{1}\right)}{R_{1} \cosh \left(R_{1}\right)-\sinh \left(R_{1}\right)} \geqslant \frac{3(C-A)}{B R_{1}^{2}} .
$$

As we note in Remark 4.4, the left-hand side of this relation is a decreasing function of $R_{1}$, and since $R_{1}>R_{1 *}>R_{\odot}$, we get together with (4.3)

$$
\frac{2}{D}>\frac{1}{D}+\frac{\sinh \left(R_{1}\right)}{R_{1} \cosh \left(R_{1}\right)-\sinh \left(R_{1}\right)} \geqslant \frac{3(C-A)}{B R_{1}^{2}} .
$$

Summarizing, due to (4.1), we obtain

$$
\frac{R_{1} \sinh \left(R_{1}\right)}{3 Q}<\frac{1}{\operatorname{coth}(1)-1}<\frac{D-1}{6}<\frac{D-1}{D} \frac{B R_{1}^{2}}{9(C-A)},
$$

which proves (4.26), meaning that $S_{0}<0$.

In order to prove that $S_{k}$ are negative, we proceed as follows. We first estimate the constants $s_{k}$ by using (4.22) and (4.23):

$$
\begin{aligned}
-R_{1}^{k-1} s_{k} & >\frac{D(D-1)(k+1) \sigma_{H}^{\prime}\left(R_{1}\right) R_{2}^{2 k+1}}{\bar{u}_{0}\left(R_{1}\right) R_{1} R_{2}^{2 k+1}((D-1) k+(k+1) D+k)+R_{1}^{2} \bar{u}_{0}^{\prime}\left(R_{1}\right) R_{2}^{2 k+1}} \\
& \geqslant \frac{(D-1)(k+1)}{(2 k+1) D \sinh \left(R_{1}\right)+Q} \frac{B R_{1}}{3(C-A)},
\end{aligned}
$$

with $Q$ as in the proof of Theorem 4.1. Given $k \geqslant 1$, it is not difficult to see that $S_{k}<0$, provided

which is equivalent to

$$
\frac{1}{R_{1}}+\frac{(D-1)\left(k^{2}+k\right)}{(2 k+1) D \sinh \left(R_{1}\right)+Q} \frac{C}{3(C-A)} \geqslant \frac{R_{1} \sinh \left(R_{1}\right)}{3 Q},
$$

$$
\frac{C}{3(C-A)} \geqslant\left[\frac{R_{1} \sinh \left(R_{1}\right)}{3 Q}-\frac{1}{R_{1}}\right] \frac{(2 k+1) D \sinh \left(R_{1}\right)+Q}{(D-1)\left(k^{2}+k\right)} .
$$

However, this last relation is satisfied by (4.4). We have thus verified that the spectrum $\sigma(\partial \Phi(0))$ is bounded away from zero in the negative half-plane, which yields in view of [26, Theorem 9.1.2] the desired result.

\section{Conclusion}

In this paper we analyze a two-phase mixture model for avascular tumor growth, when considering cell-to-cell adhesion and repulsion, and taxis due to nutrient in the tumor component. Taking advantage of the solvability of a diffraction problem related to 
the model, we show that the three-dimensional mathematical setting is equivalent to an abstract evolution equation for the sharp interface separating the tumor from the surrounding healthy tissue. This abstract setting allows us to use the parabolic theory and prove the local well-posedness of the problem.

If the rate between cell apoptosis and cell mitosis is less than the nutrient concentration in the far field tissue, then the model possesses radially symmetric steady-state solutions. For these states, the tumor is a sphere and the surrounding tissue is an annulus. Our analysis delivers precise bounds for the radius of the tumor and a formula for the exterior radius of the annulus. Using the principle of linearized stability, we also study the asymptotic behavior of arbitrary tumor domains which are initially close to a radially symmetric steady state. Particularly, if the diffusion in the tumor is faster than that in the surrounding tissue, and the regions rich in nutrients are close to the tumor boundary, then the tumor will not converge to the radially symmetric equilibrium. This comes as a completion of the two-dimensional numerical simulations in [3] where the invasive character of the tumor in the surrounding tissue is illustrated. But there is also a regime of stability, where starting close to the radially symmetric steady state, the tumor will evolve towards the latter at an exponential rate. This regime can be described, in a rather complicated way, in terms of the biophysical parameters involved in the modeling, which also stands for the complexity of this mixture model.

\section{References}

[1] Byrne, H. M. \& Chaplain, M. A. (1995) Growth of nonnecrotic tumors in the presence and absence of inhibitors. Math. Biosci. 130, 151-181.

[2] Byrne, H. M. \& Preziosi, L. (2003) Modelling solid tumour growth using the theory of mixtures. Math. Med. Biol. 20, 341-366.

[3] Cristini, V., Li, X., Lowengrub, J. \& Wise, S. M. (2009) Nonlinear simulation of solid tumor growth using a mixture model: Invasion and branching, J. Math. Biol. 58, 723-763.

[4] Cristini, V., Lowengrub, J. \& Nie, Q. (2003) Nonlinear simulation of tumor growth. J. Math. Biol. 46, 191-224.

[5] CuI, S. B. (2005) Analysis of a free boundary problem modeling tumor growth. Acta Math. Sin. Engl. Ser. 21(5), 1071-1082.

[6] Cui, S. B. \& EsCHER, J. (2007) Bifurcation analysis of an elliptic free boundary problem modelling the growth of avascular tumors. SIAM J. Math. Anal. 39(1), 210-235.

[7] CUI, S. B. \& Escher, J. (2008) Asymptotic behaviour of solutions of a multidimensional moving boundary problem modeling tumor growth. Comm. Part. Diff. Equ. 33(4), 636-655.

[8] CUI, S. B. \& Escher, J. (2009) Well-posedness and stability of a multi-dimensional tumor growth model. Arch. Ration. Mech. Anal. 191, 173-193.

[9] Cui, S. B., Escher, J. \& Zhou, F. (2008) Bifurcation for a free boundary problem with surface tension modelling the growth of multi-layer tumors. J. Math. Anal. Appl. 337(1), 443-457.

[10] Cui, S. B. \& Friedman, A. (2001) Analysis of a mathematical model of the growth of necrotic tumors. J. Math. Anal. Appl. 255, 636-677.

[11] Escher, J. \& Matioc, A.-V. (2010) Radially symmetric growth of nonnecrotic tumors. NoDEA Nonlinear Differ. Equ. Appl. 17(1), 1-20.

[12] Escher, J. \& MATIOC, A.-V. (2011) Well-posedness and stability analysis for a moving boundary problem modelling the growth of nonnecrotic tumors. Discrete Continuous Dyn. Syst. B 15(3), 573-596. 
[13] Escher, J., Matioc, A.-V. \& Matioc, B.-V. (2011a) Analysis of a Mathematical Model Describing Necrotic Tumor Growth, Lecture Notes in Applied and Computational Mechanics, Vol. 57, Springer-Verlag, New York, 237-250.

[14] Escher, J. \& Matioc, B.-V. (2011b) On the parabolicity of the Muskat problem: Wellposedness, fingering, and stability results. Z. Anal. Anwend. 30(2), 193-218.

[15] Escher, J., \& Matioc, A.-V. \& Matioc, B.-V. (2012) A generalised Rayleigh-Taylor condition for the Muskat problem. Nonlinearity 25(1), 73-92.

[16] Escher, J. \& Seiler, J. (2008) Bounded $H_{\infty}$ calculus for pseudo-differential operators and applications to the Dirichlet-Neumann operator. Trans. Amer. Math. Soc. 360(8), 3945-3973.

[17] Escher, J. \& Simonett, G. (1997a) Classical solutions for Hele-Shaw models with surface tension. Adv. Diff. Equ. 2(4), 619-642.

[18] Escher, J. \& Simonett, G. (1997b) Classical solutions of multidimensional Hele-Shaw models. SIAM J. Math. Anal. 28(5), 1028-1047.

[19] Friedman, A. \& Reitich, F. (1999) Analysis of a mathematical model for the growth of tumors. J. Math. Biol. 38, 262-284.

[20] Friedman, A. \& Reitich, F. (2000) Symmetry-breaking bifurcation of analytic solutions to free boundary problems. Trans. Amer. Math. Soc. 353, 1587-1634.

[21] Friedman, A. \& Reitich, F. (2001) On the existence of spatially patterned dormant malignancies in a model for the growth of non-necrotic vascular tumors. Math. Models Methods Appl. Sci. 9(4), 601-625.

[22] Friedman, A. \& TAO, Y. (2003) Nonlinear stability of the Muskat problem with capillary pressure at the free boundary. Nonlinear Anal. 53, 45-80.

[23] Greenspan, H. P. (1972) Models for the growth of a solid tumor by diffusion. Stud. Appl. Math. LI(4), 317-340.

[24] Greenspan, H. P. (1976) On the growth and stability of cell cultures and solid tumors. $J$. Theor. Biol. 56, 229-242.

[25] Ladyzhenskaya, O. \& Ural'tseva, N. N. (1968) Linear and Quasilinear Elliptic Equations, Mathematics in Science and Engineering, Vol. 46, Academic Press, Massachusetts.

[26] LunARdi, A. (1995) Analytic Semigroups and Optimal Regularity in Parabolic Problems, Birkhäuser, Basel, Switzerland. 